\title{
Multiconfigurational second-order perturbation study of the decomposition of the radical anion of nitromethane
}

\author{
Juan F. Arenas, ${ }^{\text {a) }}$ Juan C. Otero, Daniel Peláez, and Juan Soto \\ Department of Physical Chemistry, Faculty of Sciences, University of Málaga, \\ E-29071-Málaga, Spain \\ Luis Serrano-Andrés \\ Departamento de Química Física, Instituto de Ciencia Molecular, Universitat de València, Dr. Moliner 50, \\ Burjassot, E-46100, Valencia, Spain
}

(Received 21 November 2003; accepted 20 May 2004)

\begin{abstract}
The doublet potential energy surfaces involved in the decomposition of the nitromethane radical anion $\left(\mathrm{CH}_{3} \mathrm{NO}_{2}^{-}\right)$have been studied by using the multistate extension of the multiconfigurational second-order perturbation method (MS-CASPT2) in conjunction with large atomic natural orbital-type basis sets. A very low energy barrier is found for the decomposition reaction: $\mathrm{CH}_{3} \mathrm{NO}_{2}^{-} \rightarrow\left[\mathrm{CH}_{3} \mathrm{NO}_{2}\right]^{-} \rightarrow \mathrm{CH}_{3}+\mathrm{NO}_{2}^{-}$. No evidence has been obtained on the existence of an isomerization channel leading to the initial formation of the methylnitrite anion $\left(\mathrm{CH}_{3} \mathrm{ONO}^{-}\right)$ which, in a subsequent reaction, would yield nitric oxide (NO). In contrast, it is suggested that NO is formed through the bimolecular reaction: $\mathrm{CH}_{3}+\mathrm{NO}_{2}^{-} \rightarrow\left[\mathrm{CH}_{3} \mathrm{O}-\mathrm{N}-\mathrm{O}\right]^{-} \rightarrow \mathrm{CH}_{3} \mathrm{O}^{-}+\mathrm{NO}$. In particular, the CASSCF/MS-CASPT2 results indicate that the methylnitrite radical anion $\mathrm{CH}_{3} \mathrm{ONO}^{-}$does not represent a minimum energy structure, as concluded by using density functional theory (DFT) methodologies. The inverse symmetry breaking effect present in DFT is demonstrated to be responsible for such erroneous prediction. (C) 2004 American Institute of Physics. [DOI: 10.1063/1.1772357]
\end{abstract}

\section{INTRODUCTION}

Electron attachment to the ground state of nitromethane $\left(\mathrm{CH}_{3} \mathrm{NO}_{2}\right)$ may form a dipole-bound state or a conventional (valence-bound) anionic state. ${ }^{1,2}$ The Rydberg electron transfer collision experiments of Lecomte et al. ${ }^{2}$ indicate that both forms of nitromethane radical anion are not connected by a substantial barrier, but are readily interconverted. The dipole-bound anion would be considered as a doorway state to form the conventional anion. ${ }^{1}$

An important issue controlling the electron attachment mechanism is the dipole moment of the system. Fermi and Teller $^{3}$ predicted that a fixed (nonrotating) dipole should bind an electron when the dipole moment is larger than $1.625 \mathrm{D}$. Later, Crawford and Garrett ${ }^{4}$ established that a molecule should permanently bind an electron when its dipole moment was higher than $\approx 2.5 \mathrm{D}$. The dipole moment of neutral nitromethane $(3.46 \mathrm{D})^{5}$ is well above this lower limit. Indeed, the nitromethane radical anion $\left(\mathrm{CH}_{3} \mathrm{NO}_{2}^{-}\right)$can be formed from the neutral molecule $\left(\mathrm{CH}_{3} \mathrm{NO}_{2}\right)$ by different experimental methods: (i) electron impact, ${ }^{6,7}$ (ii) collisions with atoms in highly excited Rydberg states, ${ }^{1,2,6,8}$ or (iii) collisions with alkali atoms. ${ }^{9}$ Gruzdkov and Gupta ${ }^{10}$ have also proposed that $\mathrm{CH}_{3} \mathrm{NO}_{2}^{-}$is an intermediate in the decomposition of amine-sensitized nitromethane shocked to $12-17 \mathrm{GPa}$ peak pressures.

Both valence- and dipole-bound anionic states of $\mathrm{CH}_{3} \mathrm{NO}_{2}^{-}$have been studied by Gutsev and Bartlett ${ }^{11}$ with the density functional (DFT/B3LYP), many-body perturba-

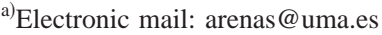

tion [MBPT(2)], and electron-attached equation-of-motion coupled-cluster (EA-EOMCC) theories. They found that the lowest dipole-bound state of nitromethane radical anion exhibits a structure very similar to neutral nitromethane, while the geometry of the valence-bound ground-state radical anion shows a tetrahedral arrangement of the nitrogen atom. Experimentally, the electron affinities (EA) related to the valence- and dipole-bound states were measured as 0.26 $\pm 0.08 \mathrm{eV}$ and $12 \pm 3 \mathrm{meV}$, respectively. ${ }^{1}$ Contradictory results were however obtained at different levels of theory. Regarding the calculation of EA for the valence-bound anion, the MBPT(2) method failed to yield a bound structure for the system, while coupled-cluster methods were found to be extremely dependent on the selection of the one-electron basis set. A usually accurate approach such as $[\operatorname{CCSD}(\mathrm{T})]$ produced errors close to $0.3 \mathrm{eV}$ even using large and diffuse basis sets. Instead, DFT/B3LYP results were preferred, yielding a value of $0.22 \mathrm{eV}$, in close agreement with experiment. In contrast, calculations on EA for the dipole-bound state were found to be converged with the diffuseness of the basis set at different levels of theory. A value of $13 \mathrm{meV}$ was obtained with the EA-EOMCCSD method, a result which nicely agrees with the experimental value, $12 \mathrm{meV} .{ }^{1}$ Later, Gutsev, Jena, and Bartlett ${ }^{12}$ studied the valence-bound methylnitrite radical anion $\left(\mathrm{CH}_{3} \mathrm{ONO}^{-}\right)$with similar methods. Two stable isomers (trans and cis) were characterized at the MBPT(2) level. A remarkable result of their work was the anomalous long distance $(1.95 \AA)$ predicted for the $\mathrm{CH}_{3} \mathrm{O}-\mathrm{NO}^{-}$bond. The computed adiabatic electron affinity 


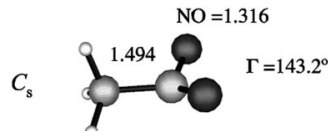

(a)

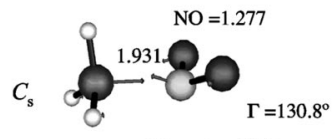

(b) $\quad \omega=559 i$

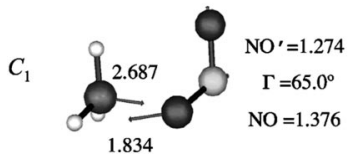

(c) $\quad \omega=1273 i$

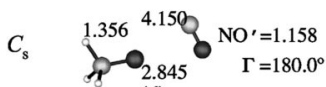

(d)

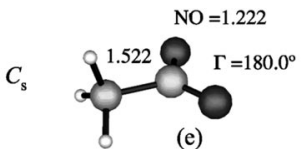

(e)
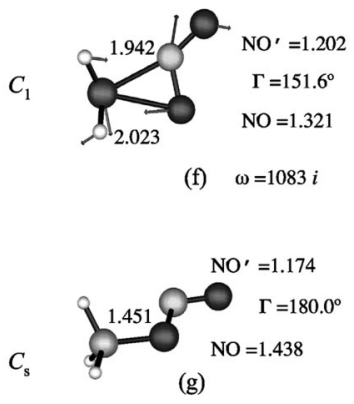

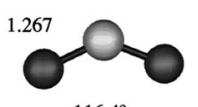

(h)

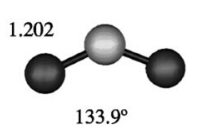

(i)
FIG. 1. Optimized geometries at the CASSCF level on the doublet and singlet potential energy surfaces of the anionic and neutral species of nitromethane. (a) Doublet minimum of the radical anion of nitromethane; (b) TS1, doublet transition state leading to $\mathrm{CH}_{3}+\mathrm{NO}_{2}^{-}$; (c) TS2, doublet transition state leading to $\mathrm{CH}_{3} \mathrm{O}^{-}+\mathrm{NO}$; (d) van der Waals type complex formed by the methoxy anion $\left(\mathrm{CH}_{3} \mathrm{O}^{-}\right)$and nitric oxide (NO); (e) singlet minimum of nitromethane; (f) singlet transition state for neutral nitro-to-nitrite isomerization; $(\mathrm{g})$ singlet minimum of trans-methylnitrite; (h) nitrite ion $\left(\mathrm{NO}_{2}^{-}\right)$; and (i) nitrogen dioxide $\left(\mathrm{NO}_{2}\right) . \Gamma$ is the ONO-C dihedral angle. The arrows in the structures correspond to the imaginary mode for the transition states. All the molecular structures in this work have been plotted with the program MacMolPlt (Ref. 33).
(0.60 eV at the DFT/B3LYP level) for $\mathrm{CH}_{3} \mathrm{ONO}$ was somewhat higher than that of $\mathrm{CH}_{3} \mathrm{NO}_{2}$.

In the present work, several processes involving the nitromethane $\left(\mathrm{CH}_{3} \mathrm{NO}_{2}\right)$ and its valence-bound radical anion $\left(\mathrm{CH}_{3} \mathrm{NO}_{2}^{-}\right)$have been studied at the CASSCF and MSCASPT2 levels of theory. Our goal was to establish accurate dissociation paths leading to the formation of $\mathrm{NO}_{2}^{-}$or $\mathrm{NO}$. Only valence-bound anionic states will be addressed. First, the dissociation channel yielding the nitrite anion and proceeding via doublet potential energy surfaces and related to the reaction

$$
\mathrm{CH}_{3} \mathrm{NO}_{2}^{-} \rightarrow \mathrm{CH}_{3}+\mathrm{NO}_{2}^{-}
$$

was studied. Second, the nitro-to-nitrite rearrangement

$$
\mathrm{CH}_{3} \mathrm{NO}_{2}^{-} \rightarrow \mathrm{CH}_{3} \mathrm{ONO}^{-}
$$

was also analyzed. This process would explain the observation of $\mathrm{O}^{-}$atoms in collisions of alkali atoms with nitromethane ${ }^{9}$ or in electron impact experiments. ${ }^{7}$ As it will be shown, no evidence of the existence of the isomerization channel toward $\mathrm{CH}_{3} \mathrm{ONO}^{-}$was obtained. In contrast, it is found that the bimolecular process yielding methylnitrite radical anion is an alternative path which actually leads to nitric oxide (NO) formation

$$
\mathrm{CH}_{3}+\mathrm{NO}_{2}^{-} \rightarrow\left[\mathrm{CH}_{3} \mathrm{O}-\mathrm{N}-\mathrm{O}\right]^{-} \rightarrow \mathrm{CH}_{3} \mathrm{O}^{-}+\mathrm{NO} \text {. }
$$

Additionally, the present study demonstrates that the molecular arrangement of $\mathrm{CH}_{3} \mathrm{ONO}^{-}$predicted by the DFT methods as an energy minimum is a spurious result obtained because of the inadequate treatment of the charge distribution at long internuclear distances.

\section{METHODS OF CALCULATION}

Generally contracted basis sets of atomic natural orbital (ANO) type obtained from $\mathrm{C}, \mathrm{N}, \mathrm{O}(14 s 9 p 4 d 3 f) / \mathrm{H}(8 s 4 p 3 d)$ primitive sets ${ }^{13}$ the so-called ANO-L basis sets, were used in all the calculations. The final results were computed using the $\mathrm{C}, \mathrm{N}, \mathrm{O}[4 s 3 p 2 d 1 f] / \mathrm{H}[3 s 2 p 1 d]$ contraction scheme, al- though smaller basis sets and the addition of more diffuse uncontracted functions were tested in order to check the convergence of the obtained results.

Calculations were initially performed at the complete active space self-consistent field (CASSCF) ${ }^{14}$ level of theory as implemented in the MOLCAS 5.4 program. ${ }^{15}$ Hereafter, $\mathrm{CAS}(\mathrm{Ne}, \mathrm{Mo})$ means the active space built by distributing $N$ electrons in $M$ orbitals. After optimization, the stationary points (minima and saddle points) were characterized by their CASSCF analytic harmonic vibrational frequencies computed by diagonalizing the mass-weighted Cartesian force constant matrix, i.e., the Hessian matrix $\boldsymbol{H}$.

Additionally, the energies of all the critical points have been recomputed with the multistate extension of the multiconfigurational second-order perturbation theory (MS-CASPT2). ${ }^{16}$ The CASSCF wave functions were therefore used as reference in the second-order perturbation treatment, freezing the $1 \mathrm{~s}$ electrons of the carbon, oxygen, and nitrogen atoms as determined in the CASSCF calculations. To minimize the contamination of the pertubated wave function by intruder states, the technique of the imaginary level shift ${ }^{17}$ has been introduced when necessary.

Whereas the theoretical treatment of dipole-bound anions represents a serious challenge for the conventional methods of quantum chemistry, the valence anions can be treated with usual methods, provided that the stability of the results with the one-electron basis set is checked. ${ }^{18}$

DFT calculations have been carried out with the B3LYP nonlocal exchange correlation functional as implemented in the GAUSSIAN 98 set of programs ${ }^{19}$ in conjunction with the $c c-p \mathrm{VDZ}$ and $c c-p \mathrm{VTZ}$ basis sets ${ }^{20}$ supplemented with diffuse functions on all the atoms.

\section{RESULTS AND DISCUSSION}

\section{A. Electronic structure of the valence-bound state of nitromethane radical anion}

The symmetry of the optimized valence-bound ground state of the nitromethane radical anion is $C_{s}$, with a tetrahedral arrangement at the nitrogen atom [Fig. 1(a)]. The occu- 
TABLE I. MS-CASPT2 energies of the doublet and singlet potential energy surfaces of nitromethane. ${ }^{\text {a }}$

\begin{tabular}{lcccc}
\hline \hline \multicolumn{1}{c}{ Geometry } & $\mathrm{wg}^{\mathrm{b}}$ & MS-CASPT2 & $\mathrm{ZPE}^{\mathrm{c}}$ & $\Delta E(\mathrm{eV})^{\mathrm{d}}$ \\
\hline Anion [Fig. 1(a)] & 0.83 & -244.62640 & 0.13 & $-0.18^{\mathrm{f}}$ \\
TS1 [Fig. 1(b)] & 0.82 & -244.58405 & 0.12 & 0.96 \\
TS2 [Fig. 1(c)] & $0.82^{\mathrm{e}}$ & $-244.58063^{\mathrm{e}}$ & 0.12 & 1.05 \\
VdW [Fig. 1(d)] & 0.84 & -244.60926 & 0.12 & 0.27 \\
$\mathrm{CH}_{3}+\mathrm{NO}_{2}^{-}$ & 0.83 & -244.59319 & 0.10 & 0.70 \\
VEA & $0.83^{\mathrm{c}}$ & $-244.60498^{\mathrm{e}}$ & 0.12 & $0.40^{\mathrm{f}}$ \\
Nitromethane [Fig. 1(e)] & 0.86 & -244.62022 & 0.14 & 0.0 \\
& $(0.86)^{\mathrm{e}}$ & $(-244.62022)^{\mathrm{e}}$ & & 3.00 \\
TS1' [Fig. 1(f)] & 0.85 & -244.50962 & 0.13 & 0.21 \\
Methylnitrite [Fig. 1(g)] & 0.86 & -244.61228 & 0.14 & 2.66 \\
$\mathrm{CH}_{3}+\mathrm{NO}_{2}$ & 0.83 & -244.52116 & 0.11 & \\
\hline \hline
\end{tabular}

${ }^{a}$ ANO-L basis sets, CAS $(15 e, 11 o)$.

${ }^{b}$ Weight of the CASSCF reference wave function (\%).

'Zero point energy in $\mathrm{eV}$.

${ }^{\mathrm{d}}$ Relative energy referred to neutral nitromethane, ZPE correction is included.

eImaginary level shift applied (LS=0.1).

fElectron affinity must be read with the sign reversed.

pied Hartree-Fock molecular orbitals of the neutral molecule in its ground state are.

$$
\begin{gathered}
1 s_{\mathrm{O}}, 1 s_{\mathrm{O}}, 1 s_{\mathrm{N}}, 1 s_{\mathrm{C}}, 2 s_{\mathrm{O}}, 2 s_{\mathrm{O}}, \sigma_{\mathrm{CH}}, 2 s_{\mathrm{N}}, \sigma_{\mathrm{NO}}, \pi_{\mathrm{NO}}, \sigma_{\mathrm{NO}}, \\
\sigma_{\mathrm{CH}}, \sigma_{\mathrm{CH}}, \mathrm{n} \sigma_{\mathrm{O}}, \sigma_{\mathrm{CN}}, \mathrm{n} \pi_{\mathrm{O}}
\end{gathered}
$$

where the subscripts indicate the dominant character of each orbital.

The selected molecular orbitals of the radical anion included in the active space were those arising from all the molecular valence orbitals plus their corresponding antibonding orbitals, with the exception of the $2 s$ orbitals on oxygen and the three $\sigma\left(\mathrm{CH}_{3}\right)$ orbitals. Therefore, the active space comprises 11 molecular orbitals and 15 electrons. This choice is coincident with the previous one we used in the neutral molecule, ${ }^{21,22}$ which was able to prevent the symmetry breaking of the wave function. At the employed levels of theory, the extra electron of the valence anion occupies the $\pi_{\mathrm{NO}}^{*}\left(A^{\prime}\right)$ orbital which corresponds to the LUMO orbital of the neutral molecule.

At the CASSCF level, the energy of radical anions tends to be overestimated with respect to that of the corresponding neutral molecules, although the results represent, however, well-localized solutions for valence-bound states in anions. $^{23-25}$ Therefore, the usual computational strategy of obtaining optimized geometries at the CASSCF level and performing fixed-geometry CASPT2 calculations can be used safely, considering that has proved its accuracy before. ${ }^{26}$ Prior to the discussion of the reaction paths leading to the breaking of the $\mathrm{C}-\mathrm{N}$ bond in nitromethane radical anion or to its isomerization to methylnitrite radical anion $\left(\mathrm{CH}_{3} \mathrm{ONO}^{-}\right)$, all stationary points on the doublet potential energy surfaces are presented (Fig. 1). For the sake of comparison, the geometries of the corresponding neutral compounds are also included.

The CASPT2 energies of all the species studied in this work are collected in Table I, as well as the zero-point energy (ZPE) correction to each structure. The electron affinity (EA) and vertical electron affinity (VEA) of $\mathrm{CH}_{3} \mathrm{NO}_{2}^{-}$are computed according to Eqs. (1) and (2), respectively,

$$
\begin{aligned}
\mathrm{EA}= & E_{\mathrm{el}}(N)+\mathrm{ZPE}_{N}-E_{\mathrm{el}}(A)-\mathrm{ZPE}_{A}=\Delta E_{\mathrm{el}}+\Delta E_{\mathrm{nuc}}, \\
\mathrm{VEA} & =E_{\mathrm{el}}(N)+\mathrm{ZPE}_{N}-E_{\mathrm{el}}\left(N^{-}\right)-\mathrm{ZPE}_{N^{-}} \\
& =\Delta E_{\mathrm{el}}+\Delta E_{\mathrm{nuc}},
\end{aligned}
$$

where $N$ and $A$ refer to the minimum energy geometries of the neutral and anion species, respectively, and $E_{\mathrm{el}}\left(N^{-}\right)$represents the electronic energy of the anion computed at the geometry of the neutral molecule [Fig. 1(e)]

In order to compute the ZPE correction to $N^{-}$(a nonstationary point), the vibrational frequencies are obtained after the energy gradient, rotations, and translations were projected out of the geometrical Hessian according with Eq. $(3)^{27}$

$$
\boldsymbol{H}^{P}=(1-\boldsymbol{P}) \boldsymbol{H}(1-\boldsymbol{P}),
$$

where $\boldsymbol{H}$ is the $3 N \times 3 N$ Hessian matrix, $\boldsymbol{H}^{P}$ is the projected force constant matrix, and $\boldsymbol{P}$ is the projector, a $3 N \times 3 N$ matrix, which in turn is the sum of seven matrices built from the unit vector that points along the mass-weighted gradient and the six $3 N$-dimensional unit vectors that correspond to infinitesimal rotations and translations. ${ }^{28}$ The discussed energy differences will include hereafter the vibrational zero-point correction.

As displayed in Table I, a negative vertical electron affinity for nitromethane of $-0.40 \mathrm{eV}$ is computed at the MSCASPT2 level. The attachment of an electron to the neutral system is therefore not favored in these conditions. In contrast, the "adiabatic" result EA yields a positive value 0.18 $\mathrm{eV}$ and, consequently, the valence anion is proved to be more stable than the neutral compound. The computed value is in good agreement with the measured datum $0.26 \pm 0.08 \mathrm{eV}{ }^{1}$ Unlike previous $a b$ initio results $[\mathrm{MBPT}(2)$ and $\operatorname{CCSD}(\mathrm{T})$, see the discussion in the introduction] the MS-CASPT2 value proved to be stable with respect to changes in the ANO one-electron basis set. For instance, removing polarization functions from the basis sets ( $f$ on $\mathrm{C}, \mathrm{N}$, and $\mathrm{O}$ ) just reduces the EA to $0.16 \mathrm{eV}$. On the other hand, several tests were performed in which the diffuseness of the basis functions 


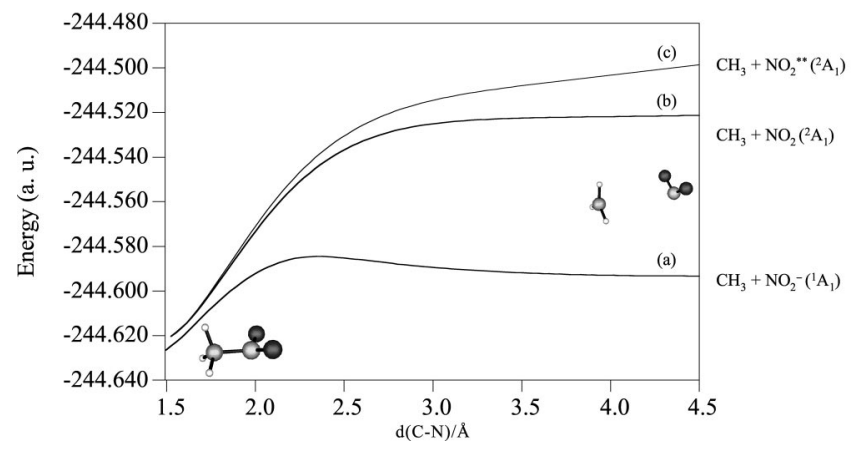

FIG. 2. CASPT2 potential energy curves of the valence-bound state of the nitromethane radical anion $\left({ }^{2} A^{\prime}\right)$ and neutral nitromethane $\left({ }^{1} A^{\prime}\right)$ leading to dissociation into $\mathrm{CH}_{3}$ and $\mathrm{NO}_{2}^{-}$or $\mathrm{NO}_{2}$, respectively. (a) Doublet potential energy surface, the starting point is the minimum of the anion and the final point is the arrangement corresponding to noninteracting methyl radical and nitrite ion fragments; (b) singlet potential energy surface, the starting point is the minimum of the neutral molecule and the final point is the arrangement corresponding to non-interacting methyl radical and nitric oxide fragments; and (c) singlet potential energy surface, the starting point is the minimum of the neutral molecule and the final point is the arrangement corresponding to noninteracting methyl radical and nitrite ion fragments; however, the electronic structure of the $\mathrm{NO}_{2}$ fragment corresponds to the radical not to the anion.

was increased, just with the purpose of checking the stability of the results. When uncontracted diffuse functions were added at the charge centroid of the molecule, different behavior was found for the states of the anion. Starting with exponents of the primitives similar to those typically used to compute Rydberg states ${ }^{26}$ (from 0.024 to 0.0006 ) and scaling by different factors: $0.5,0.1$, and 0.01 , the stabilization of the MS-CASPT2 results could be analyzed. ${ }^{18}$ The lowest solution, clearly the dipole-bound state, proves to be extremely dependent of the employed exponents. On the contrary, the valence-bound anionic ground state remains stable within $0.05 \mathrm{eV}$. The use of flexible and balanced ANO-type basis sets is behind this behavior. They have proved to be accurate to deal with valence-bound anionic states in different systems. ${ }^{23-25}$

\section{B. Dissociation of the radical anion into methyl radical $\left(\mathrm{CH}_{3}\right)$ and nitrite ion $\left(\mathrm{NO}_{2}^{-}\right)$}

Figure 2 displays the CASPT2 potential energy curves $\left(C_{s}\right.$ symmetry) for the dissociation of the ground electronic states of the radical anion and the neutral molecule, yielding as final products $\mathrm{NO}_{2}^{-}$plus $\mathrm{CH}_{3}$ in the doublet state [Fig. 2(a)] and $\mathrm{NO}_{2}$ plus $\mathrm{CH}_{3}$ on the singlet surface [Fig. 2(b)], respectively. These curves have been obtained via interpolation between the stationary geometries of Figs. 1(a) or 1(e) and those corresponding to the isolated fragments, $\mathrm{NO}_{2}^{-}$ [Fig. 1(h)] or $\mathrm{NO}_{2}$ [Fig. 1(i)], and $\mathrm{CH}_{3}$. Additionally, the following restrictions have been imposed: (1) In all the interpolations the final $\mathrm{C}-\mathrm{N}$ distance is $4.5 \AA$. This choice is based on two criteria fulfilled in the ground state of the neutral molecule: (i) there is no significant gradient of energy at this distance and (ii) the charges of the resulting fragments are already zero. (2) The geometry of $\mathrm{CH}_{3}$ at the end of all the interpolations is always the optimized structure of its ground state as obtained by performing a ROHF calculation,
CAS $(1 e, 1 o)$. (3) The final dihedral angle between the respective planes containing the $\mathrm{CH}_{3}$ and $\mathrm{NO}_{2}$ radicals is $90^{\circ}$.

A small exit barrier for $\mathrm{CH}_{3} \mathrm{NO}_{2}^{-}$decomposition into $\mathrm{CH}_{3}$ and $\mathrm{NO}_{2}^{-}\left({ }^{1} A_{1}\right)$ is observed in the interpolation curve [Fig. 2(a)]. In contrast, the dissociation of the neutral compound [Fig. 2(b)] takes place without any exit barrier. Actually, we were able to locate the doublet transition state [TS1, Fig. 1(b)] leading to the dissociation of $\mathrm{CH}_{3} \mathrm{NO}_{2}^{-}$into $\mathrm{CH}_{3}$ and $\mathrm{NO}_{2}^{-}$(ground states of methyl radical and nitrite ion). TS1 is placed $18 \mathrm{kcal} / \mathrm{mol}$ above the minimum of $\mathrm{CH}_{3} \mathrm{NO}_{2}^{-}$ and $14 \mathrm{kcal} / \mathrm{mol}$ above the computed vertical electron affinity (Table I). Therefore, the valence-bound anion is stabilized against dissociation.

In addition, the heterolytic $\mathrm{C}-\mathrm{N}$ bond-breaking of nitromethane has been studied [Fig. 2(c)] by forcing the dissociation fragments to reach the methyl cation $\left(\mathrm{CH}_{3}^{+}\right)$and nitrite anion $\left(\mathrm{NO}_{2}^{-}\right)$at their respective geometries. The final electronic structure for these molecular arrangements corresponded instead to the neutral fragments. Accordingly, we consider that heterolytic dissociation in the gas phase has to be ruled out.

\section{Does an isomerization channel for $\mathrm{CH}_{3} \mathrm{NO}_{2}^{-}$ to $\mathrm{CH}_{3} \mathrm{ONO}^{-}$exist?}

The first question rising in this section is, Does the nitrite anion isomer $\mathrm{CH}_{3} \mathrm{ONO}^{-}$actually exist? DFT/B3LYP results [Fig. 3(d)] seem to support the hypothesis, yielding an optimized minimum structure with an anomalous long O-NO distance $(1.890 \AA)$, similar to that obtained with previous EA-EOMCCSD and MBPT(2) results. ${ }^{12}$

In contrast, the structure optimized at the CASSCF level [Fig. 1(d)] should not be considered as the energy minimum of a conventional molecule, but more likely to be a van der Waals-type complex. Several features of the system support this assumption: (i) the distance between the centers of both fragments is quite large $(\sim 4 \AA)$; (ii) the vibrational analysis shows very low frequencies for several modes; and (iii) there is a perfect charge separation between both fragments (NO and $\mathrm{CH}_{3} \mathrm{O}$ ), i.e., $\mathrm{NO}$ is neutral while the methoxy fragment has charge -1 .

In order to solve this apparent discrepancy, we have computed the dissociation curve of the trans-methylnitrite "anion" $\left[\mathrm{CH}_{3} \mathrm{ONO}^{-}\right.$, Fig. 3(d)] at the DFT/ B3LYP/aug- $c c-p$ VDZ level (Fig. 4). The electronic energy increases as the molecule approaches the dissociation channel, that is, there is no tendency to reach an asymptotic limit. In the inset of the same figure, the charge on the NO fragment is represented along the interpolation path. An unphysical behavior is observed: The emerging products are absurdly charged at the dissociation limit $\left(\mathrm{CH}_{3} \mathrm{O}^{\delta-}+\mathrm{NO}^{\gamma-}\right.$, $\delta+\gamma=+1)$. In fact, both observations are two well-known flaws of the DFT methods due to the so-called inverse symmetry breaking effect, a term coined by Bally and Sastry ${ }^{29}$ who reported it. The name of this spurious effect comes from the fact that the charge localization of the density functional methods contrasts with that obtained from some selfconsistent-field results (SCF) methods when symmetry 


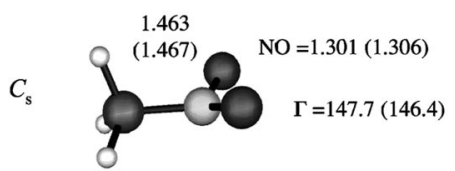

(a) $(0.0 \mathrm{eV}) ; E A_{\text {ad }}=0.38 \mathrm{eV}$

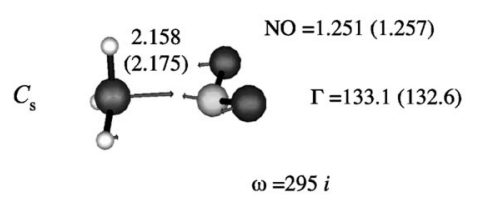

(b) $(0.83 \mathrm{eV})$

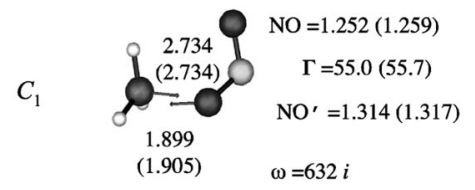

(c) $\quad(1.18 \mathrm{eV})$

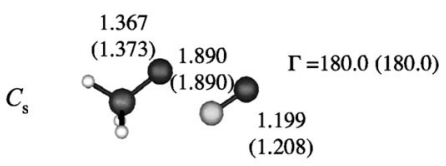

(d) $\quad(-0.10 \mathrm{eV})$
FIG. 3. Optimized geometries on the doublet potential energy surfaces of the nitromethane radical anion at the DFT/B3LYP/aug- $c c-p$ VTZ level. (a) Doublet minimum of the radical anion of nitromethane; (b) $\mathrm{TS} 1_{\mathrm{DFT}}$, doublet transition state leading to $\mathrm{CH}_{3}+\mathrm{NO}_{2}^{-}$; (c) $\mathrm{TS} 2_{\mathrm{DFT}}$, doublet transition state for the bimolecular reaction [Eq. (5)]; and (d) "minimum" of transmethylnitrite anion. Relative energies (in electron volts) referred to the minimum of the radical anion. In parentheses, DFT/B3LYP/aug- $c c-p \mathrm{VDZ}$ values. breaking is present. While the symmetry breaking deficiency of the SCF methods localizes the charge in one of the dissociating fragments, inverse symmetry breaking favors an incorrect delocalized electron density distribution. Xie et al. ${ }^{30}$ have pointed out that this effect may be an intrinsic defect of the non-Hartree-Fock exchange functionals, yielding a physically incorrect picture of the potential energy surfaces. Inverse symmetry breaking has been reported as a common phenomenon appearing in the dissociation of radical ions with two equivalent fragments. ${ }^{29,30}$ Recently, we have found the effect in the dissociation of neutral nitromethane into methyl radical and nitric oxide. ${ }^{22}$ In fact, it is well known that present-day functionals yield erroneous results for highly charged systems, charge transfer situations, molecules including atoms with high $Z$, or for cases which are not well described by a single reference wave function. ${ }^{31,32}$

Once established that the methylnitrite radical anion DFT structure represents a spurious solution, we have carefully searched on the doublet potential energy surface in the expected region of the nitromethane-to-methylnitrite radical anion "isomerization." The only transition structure found in this domain of the potential energy surface was that we labeled TS2 [Fig. 1(c)]. This geometry is found at the DFT level as well [TS2 $2_{\text {DFT }}$, Fig. 3(c)], and it is quite similar both

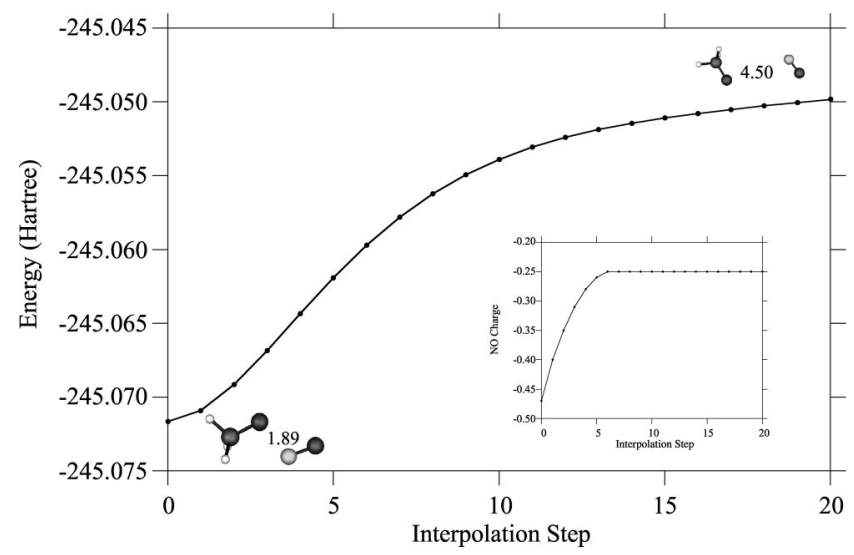

FIG. 4. Interpolation curve at the DFT/B3LYP/aug-cc- $p$ VDZ level leading from cis-methylnitrite to dissociation into methoxy anion $\left(\mathrm{CH}_{3} \mathrm{O}^{-}\right)$and nitric oxide (NO). The charge on the NO fragment along the interpolation path is represented in the inset. energetically and structurally to TS2. Starting at TS2 2 DFT, Fig. 5 displays an intrinsic reaction coordinate profile computed at the DFT/B3LYP/aug- $c c-p$ VDZ level. The forward direction would lead to formation of the methoxy anion, $\mathrm{CH}_{3} \mathrm{O}^{-}$plus $\mathrm{NO}$, while the reverse path would end at the dissociation products $\left(\mathrm{CH}_{3}+\mathrm{NO}_{2}^{-}\right)$. In the same figure, the CASPT2 linear interpolation is represented as an inset, starting at the CASSCF TS2 transition state [Fig. 1(c)] and leading to the van der Waals type complex [Fig. 1(d)], precursor of the dissociation $\mathrm{CH}_{3} \mathrm{O}^{-}+\mathrm{NO}$. Consequently, the previous results indicate that TS2 is the transition state for the bimolecular reaction

$$
\mathrm{CH}_{3}+\mathrm{NO}_{2}^{-} \rightarrow\left[\mathrm{CH}_{3}-\mathrm{O}-\mathrm{NO}\right]^{-} \rightarrow \mathrm{CH}_{3} \mathrm{O}^{-}+\mathrm{NO} \text {. }
$$

We must conclude that methylnitrite radical anion $\mathrm{CH}_{3} \mathrm{ONO}^{-}$is not stable and represents a transition state structure. Previous characterizations performed for the same species at the MBPT(2) level of theory should be questioned because of the intrinsic inaccuracy of the methodology in this type of systems. For instance, the nitromethane radical anion is not bound at all at the MBPT(2) level, and the errors in the adiabatic electron affinities are larger than $0.4 \mathrm{eV}$.

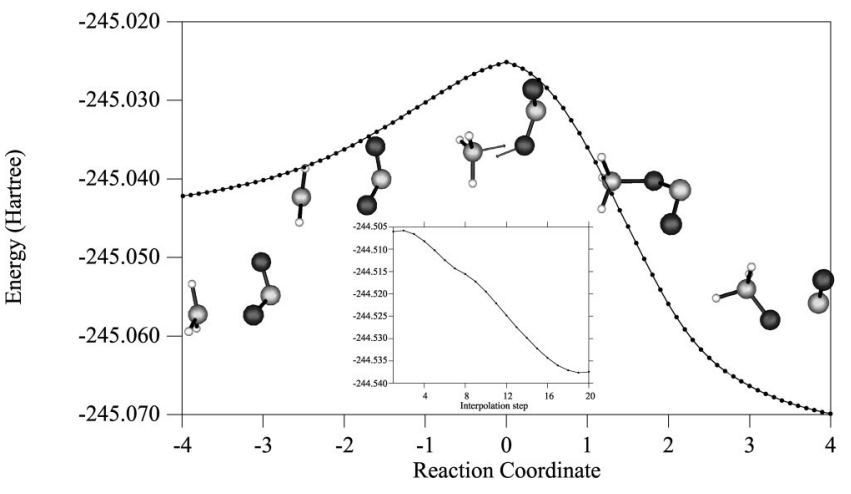

FIG. 5. IRC starting at the TS2 $\mathrm{DFT}$ transition state (DFT/B3LYP/aug- $c c-p$ VDZ). Forward direction ends at the methylnitrite "minimum." Backward direction ends at the dissociation products. The energy profile for the TS2 to VdW geometries at the CASPT2 level is represented in the inset. 


\section{SUMMARY}

The ground state of nitromethane radical anion is found to be of ${ }^{2} A^{\prime}$ symmetry, and corresponds to the attachment of the extra electron to the $\pi^{*}\left(\mathrm{NO}_{2}\right)$ molecular orbital. The computed adiabatic electron affinity is positive at the MS-CASPT2 level $0.18 \mathrm{eV}$ in accordance with the experimental data $0.26 \mathrm{eV}$ and in contrast with previous $a b$ initio results.

The dissociation and "isomerization" channels for the decomposition of the valence-bound ground state of nitromethane radical anion have been studied at the CASSCF, MS-CASPT2, and DFT/B3LYP levels of theory. A rather low activation energy for the dissociation path leading to formation of methyl radical $\left(\mathrm{CH}_{3}\right)$ and nitrite ion $\left(\mathrm{NO}_{2}^{-}\right)$was computed. A transition state for this channel [TS1, Fig. 1(b)] has been characterized. On the other hand, it is demonstrated that methylnitrite radical anion $\left(\mathrm{CH}_{3} \mathrm{ONO}^{-}\right)$does not represent a stable solution, so that, it is expected that no isomerizarion channel exists connecting nitromethane radical anion to $\mathrm{CH}_{3} \mathrm{ONO}^{-}$. In contrast, the two fragments emerging from dissociation $\left(\mathrm{CH}_{3}+\mathrm{NO}_{2}^{-}\right)$are shown to react via a bimolecular mechanism [TS2, Fig. 1(c)] to give methoxy anion $\left(\mathrm{CH}_{3} \mathrm{O}^{-}\right)$and nitric oxide (NO). The energy barrier for this process is about $20 \mathrm{Kcal} / \mathrm{mol}$ above the reacting fragments. The general picture of the processes is plotted below in Scheme I:

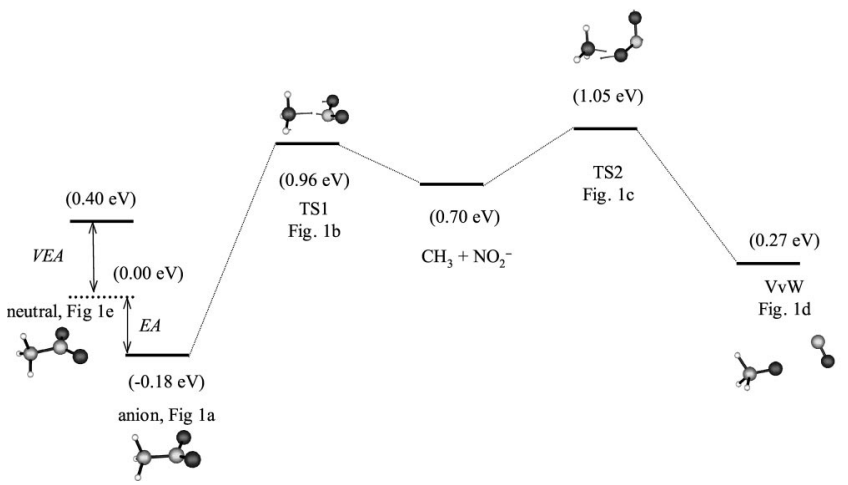

\section{ACKNOWLEDGMENTS}

This research has been supported by the Ministerio de Ciencia y Tecnología (Project BQU2000-1353). The authors thank D. R. Larrosa for the technical support in running the calculations and SCAI (University of Málaga) for use of an Origin 2000 SGI computer.
${ }^{1}$ R. N. Compton, H. S. Carman, C. Desfrançois, H. Abdoul-Carime, J. P. Schermann, J. Hendricks, S. A. Lyapustina, and K. H. Bowen, J. Chem. Phys. 105, 3472 (1996).

${ }^{2}$ F. Lecomte, S. Carles, C. Defrançois, and M. A. Johnson, J. Chem. Phys. 113, 10973 (2000).

${ }^{3}$ F. Fermi and E. Teller, Phys. Rev. 72, 399 (1947).

${ }^{4}$ O. H. Crawford and W. R. Garrett, J. Chem. Phys. 66, 4968 (1977).

${ }^{5}$ R. D. Nelson, Jr., D. R. Lide, Jr., and A. A. Maryott, Natl. Stand. Ref. Data Ser. (U.S., Natl. Bur. Stand.) 10, (1967).

${ }^{6}$ J. A. Stockdale, F. J. Davis, R. N. Compton, and C. E. Klots, J. Chem. Phys. 60, 4279 (1974).

${ }^{7}$ I. C. Walker and M. A. D. Fluendy, Int. J. Mass. Spectrom. 205, 171 (2001).

${ }^{8}$ R. N. Compton, P. W. Reinhardt, and C. D. Cooper, J. Chem. Phys. 68, 4360 (1978)

${ }^{9}$ R. F. M. Lobo, A. M. C. Moutinho, K. Lacmann, and J. Los, J. Chem. Phys. 95, 166 (1991).

${ }^{10}$ Y. A. Gruzdkov and Y. M. Gupta, J. Phys. Chem. A 102, 2322 (1998); Y. A. Gruzdkov and Y. M. Gupta, ibid. 102, 8325 (1998).

${ }^{11}$ G. L. Gutsev and R. J. Bartlett, J. Chem. Phys. 105, 8785 (1996).

${ }^{12}$ G. L. Gutsev, P. Jena, and R. J. Bartlett, J. Chem. Phys. 110, 403 (1999).

${ }^{13}$ P.-O. Widmark, P.-A. Malmqvist, and B. O. Roos, Theor. Chim. Acta 77, 291 (1990).

${ }^{14}$ Roos, B. O. in Advances in Chemical Physics; Ab Initio Methods in Quantum Chemistry II, edited by K. P. Lawley (Wiley, Chichester, England, 1987), Chap. 69, p. 399.

${ }^{15}$ K. Andersson, M. Barysz, A. Bernhardsson et al., MOLCAS VERSION 5.4 (Lund University, Sweden, 2002).

${ }^{16}$ J. Finley, P.-A. Malmqvist, B. O. Roos, and L. Serrano-Andrés, Chem. Phys. Lett. 288, 299 (1998).

${ }^{17}$ N. Forsberg and P.-A. Malmqvist, Chem. Phys. Lett. 274, 196 (1997).

${ }^{18}$ J. Simons and K. D. Jordan, Chem. Rev. (Washington, D.C.) 87, 535 (1987).

${ }^{19}$ M. J. Frisch, G. W. Trucks, H. B. Schlegel et al., GAUSSIAN 98, Revision A.7, Gaussian, Inc., Pittsburgh, PA, 1998.

${ }^{20}$ T. H. Dunning, Jr., J. Chem. Phys. 90, 1007 (1989).

${ }^{21}$ J. F. Arenas, J. C. Otero, D. Peláez, and J. Soto, J. Chem. Phys. 119, 7814 (2003).

${ }^{22}$ J. F. Arenas, S. P. Centeno, I. López-Tocón, D. Peláez, and J. Soto, J. Mol. Struct.: THEOCHEM 630, 17 (2003).

${ }^{23}$ M. Rubio, M. Merchán, E. Ortí, and B. O. Roos, J. Phys. Chem. 99, 14980 (1995).

${ }^{24}$ R. Pou-Amérigo, L. Serrano-Andrés, M. Merchán, E. Ortí, and N. Forsberg, J. Am. Chem. Soc. 122, 6067 (2000).

${ }^{25}$ L.-M. Frutos, O. Castaño, and M. Merchán, J. Phys. Chem. A 107, 5472 (2003).

${ }^{26}$ B. O. Roos, M. P. Fülscher, P.-^. Malmqvist, L. Serrano-Andrés, K. Pierloot, and M. Merchán, Adv. Chem. Phys. 93, 219 (1996).

${ }^{27}$ J. F. Arenas, J. I. Marcos, I. López-Tocón, J. C. Otero, and J. Soto, J. Chem. Phys. 113, 2282 (2000).

${ }^{28}$ S. Califano, Vibrational States (Wiley-Interscience, Bristol, 1976).

${ }^{29}$ T. Bally and G. N. Sastry, J. Phys. Chem. A 101, 7923 (1997).

${ }^{30}$ Y. Xie, H. F. Schaefer III, X.-Y. Fu, and R.-Z. Liu, J. Chem. Phys. 111, 2532 (1999).

${ }^{31}$ E. R. Davidson, Int. J. Quantum Chem. 69, 241 (1998).

${ }^{32}$ D. Tozer, R. Amos, N. Handy, B. O. Roos, and L. Serrano-Andrés, Mol. Phys. 97, 859 (1999).

${ }^{33}$ B. M. Bode and M. S. Gordon, J. Mol. Graphics Modell. 16, 133 (1999). 